COMECHINGONIA. Revista de Arqueología. Vol. 22, n 1. Primer semestre de 2018. ISSN: 0326-7911

COMITÉ EDITORIAL

DIRECTOR

SEBASTIÁN PASTOR (CITCA-CONICET-Catamarca)

CO-EDITORA

ANDREA RECALDE (CEH-IEH-CONICET-UNC-Córdoba)

CONSEJO ASESOR

JESÚS ADÁNEZ PAVÓN (UNIVERSIDAD COMPLUTENSE-Madrid)

J. ROBERTO BÁRCENA (INCIHUSA-CONICET-UNCU-Mendoza)

LUIS F. BATE (ENAH-México)

LUIS BORRERO (IMHICIHU-CONICET-Buenos Aires)

FELIPE CRIADO BOADO (INCIPIT-CSIC-Santiago de Compostela)

LEONARDO GARCÍA SANJÚAN (U. DE SEVILLA-Sevilla)

GUILLERMO MENGONI GOÑALONS (ICA-UBA-CONICET-Buenos Aires)

AXEL NIELSEN (INAPL-CONICET-Buenos Aires)

GUSTAVO POLITIS (INCUAPA-CONICET-UNCPB-Olavarría)

MYRIAM TARRAGÓ (M. ETNOGRÁFICO-UBA-CONICET-Buenos Aires)

HUGO YACOBACCIO (ICA-UBA-CONICET-Buenos Aires)

EVALUADORES PARA ESTE NÚMERO

Federico Bobillo (ISES-CONICET); María F. Bugliani (IDECU-UBA-CONICET); Rafael

Curtoni (INCUAPA-CONICET-UNCPBA); Violeta Di Prado (FCNyM-UNLP-CONICET);

Anabel Feely (IDECU-UBA-CONICET); Fernanda Falabella (Dpto. de Antropología, Universidad de Chile); Emilia Iucci (FCNyM-UNLP-CONICET ); Jimena Oria (CADICCONICET); María C. Páez (CONICET-FCNyM-UNLP); Valeria Palamarzuck (IDECUUBA-CONICET); Rafael Paunero (FCNyM-UNLP); José Porto López (LARBO-UNMDP); Cristina Prieto Olavarría (IANIGLA-CONICET); Federico Restifo (CONICET-FFyH-UBA);

Camila Riera Soto (Universidad Católica del Norte, Chile); Julián Salazar (CEH-IEHCONICET-UNC); M. Lorena Sanhueza Riquelme (Dpto. de Antropología, Universidad de Chile); Marina Sprovieri (CONICET-FCNyM-UNLP); Emily Stovel (El Zócalo Plaza Visitor and Event Center, Sandoval County Office of Economic Development, Bernalillo); Nuria Sugrañes (IANIGLA-CONICET); Veronika Szilágyi (Nuclear Analysis and Radiography Department, Centre for Energy Research, Hungarian Academy of Sciences); Cristian Vitry (UNSa); Verónica Williams (IDECU-UBA-CONICET); Federico Wynveldt (CONICETFCNyM-UNLP); M. Amalia Zaburlin (CREA-FHyCS-UNJu).

Dirección postal: Miguel C. del Corro 308, (5000) Córdoba - Argentina

Correo electrónico: revistacomechingonia@gmail.com

Web: http//www.comechingonia.com 

COMECHINGONIA. Revista de Arqueología. Vol. 22, n 1. Primer semestre de 2018. ISSN: 0326-7911

\section{Índice}

Presentación

Dossier: "Problemáticas y abordajes metodológicos en los estudios petrográficos de cerámica arqueológica".

1. Presentación.

Por: Lucas Pereyra Domingorena y Verónica Puente

2. Un volcán en la cerámica: indicios de producción en el altiplano de Isluga,

Tarapacá-Chile.

Por: Isabelle Druc y Mauricio Uribe Rodríguez

3. Una puesta al día sobre la variación petrográfica y química del estilo cerámico Inca Pacajes.

Por: Nicolás Larcher y M. Beatriz Cremonte

4. Elecciones tecnológicas y prácticas alfareras en el tambo Costa de Reyes $\mathrm{N}^{\mathrm{o}}$

5 (Tinogasta, Catamarca): aproximaciones petrográficas.

Por: Sergio Vera y Guillermo de la Fuente

5. Tecnología cerámica a través de la petrografía en la Quebrada de Miriguaca (Antofagasta de la Sierra, Catamarca) durante el Período Tardío.

Por: Leticia Gasparotti

6. Nuevas evidencias en relación a la selección de materiales cerámicos para

formar parte de los contextos funerarios. PP9-III y PP13-I como casos de estudio (ca. 1330-1000 AP) (Antofagasta de la Sierra, Catamarca).

Por: Vanesa Juárez

7. Prácticas de manufactura cerámica en el oeste riojano durante el Período

Tardío (ca. siglos XIII-XVII AD). Aportes desde los estudios petrográficos sobre el estilo Sanagasta/ Angualasto de la Tambería de Guandacol.

Por: Sebastián Carosio

8. Evidencias arqueológicas de los siglos VII a XII AD en el asentamiento de Moreta (Puna de Jujuy, Argentina).

Por: M. Josefina Pérez Pieroni y Carlos Angiorama

9. Una aproximación petrográfica a los modos de hacer y la circulación alfarera en el Área Valliserrana del Noroeste Argentino durante los últimos siglos del primer milenio D.C.

Por: Lucas Pereyra Domingorena y Verónica Puente

\section{Artículos}

1. Nuevos datos en relación a la arqueología del valle de Lerma, provincia de

Salta, Argentina.

Por: Cecilia Mercuri

2. Aplicación de los drones en diversos contextos arqueológicos y en casos de reconstrucción geoarqueológica.

Por: M. Marta Sampietro Vattuone y José Peña Monné 
3. Ritualidad y espacialidad andina a través de enfoques etnográficos y arqueológicos. Confluencias cosmogónico-ontológicas en la constitución de espacios sagrados entre El Shincal de Quimivil y ceremonias actuales de origen cusqueño.

Por: Marco Giovannetti

4. El paisaje rupestre de Cerro Colorado (provincia de Córdoba, Argentina): detectando otros sentidos, otras corporalidades.

Por: Luis Tissera 
COMECHINGONIA. Revista de Arqueología. Vol. 22, n 1. Primer semestre de 2018. ISSN: 0326-7911

DOSSIER

\title{
PROBLEMÁTICAS Y ABORDAJES METODOLÓGICOS EN LOS ESTUDIOS PETROGRÁFICOS DE CERÁMICA ARQUEOLÓGICA.
}

\section{PROBLEMS AND METHODOLOGICAL APPROACHES IN THE PETROGRAPHIC STUDIES OF ARCHAEOLOGICAL CERAMICS.}

\author{
Lucas Pereyra Domingorena ${ }^{1}$ y Verónica Puente ${ }^{2}$ \\ ${ }^{1}$ IDECU, UBA - CONICET. Moreno 350, (1091) Buenos Aires, Argentina, \\ lucasdomingorena@gmail.com; \\ 2 CONICET, LARBO - UNMDP. Av. Juan B. Justo 2550, (7600) Mar del Plata, Argentina, \\ vpuente78@yahoo.com.ar
}

Este dossier especial es el resultado de la compilación de artículos presentados como ponencias en el simposio Problemáticas y abordajes metodológicos en los estudios petrográficos de cerámica arqueológica, que se desarrolló en el XIX Congreso Nacional de Arqueología Argentina entre el 8 y 12 de agosto de 2016 bajo los auspicios de la Universidad Nacional de Tucumán. Estos trabajos se corresponden a investigaciones de los Andes Centro-Sur que utilizan la petrografía cerámica para generar información sobre las sociedades etnográficas y del pasado.

La petrografía es una rama de la geología que se ocupa del estudio e investigación de las rocas y los minerales a través de sus propiedades ópticas observadas por medio de un microscopio de polarización. La aplicación del método petrográfico a los estudios cerámicos sudamericanos -arqueológicos y etnográficos- se remontan a los trabajos pioneros de Sigvald Linné en la década de 1920, los cuales permitieron inferir comportamientos técnicos y tradiciones alfareras combinando hábilmente esta metodología geológica para resolver problemáticas antropológicas. Esa relación entre diferentes ciencias implicó una fecunda innovación para los estudios cerámicos posteriores.

En 1966, los puntos evaluados por la Convención Nacional de Antropología para el estudio tecnológico cerámico fueron coherentes con las investigaciones llevadas a cabo por Anna Shepard en EEUU desde mediados de la década de 1950. Estas indagaciones propiciaban examinar la composición de las pastas cerámicas con observación microscópica, de manera que fueron arrojando luz sobre prácticas, tradiciones y gustos implicados en la 
manufactura de las vasijas. Parte de su obra fue traducida al castellano y publicada en el volumen de la mencionada convención, lo cual facilitó su difusión en los ámbitos académicos argentinos.

Durante las décadas de 1960 y 1970, aunque estaba implícito entre los postulados de la Convención Nacional de Antropología el uso de una metodología petrográfica, las tipologías de varias regiones del Noroeste argentino siguieron presentando solamente descripciones de pastas elaboradas a partir de observaciones macroscópicas. Dos excepciones fueron los trabajos realizados en Quebrada del Toro y Yavi Chico, aunque sin especificarse aún los parámetros analíticos implementados. Pero es hacia finales de la década de 1970 cuando se incorporó por primera vez el análisis petrográfico detallado al estudio alfarero. Es a través de las descripciones realizadas por César Cortelezzi a instancia de Myriam Tarragó, quien investigaba la cerámica del área de San Pedro de Atacama, como se distinguieron diferentes composiciones petrográficas en los tipos cerámicos estudiados.

Posteriormente, en la década de 1990 los trabajos sistemáticos de Beatriz Cremonte incorporan definitivamente la metodología petrográfica a los estudios cerámicos en la arqueología argentina. Cabe mencionar también la renovación de las perspectivas teóricometodológicas implementadas en la región Pampeana por María Isabel González de Bonaveri y su equipo de colaboradores, quienes generaron un programa de investigación que contempló el análisis petrográfico en la comparación de muestras arqueológicas y experimentales.

En la actualidad, el estudio petrográfico de las pastas cerámicas arqueológicas es una herramienta fundamental en la arqueología argentina, como lo demuestra el incremento considerable de las investigaciones relacionadas con la temática, que se refleja en becas, tesis doctorales y publicaciones referentes a distintas regiones del país. Esto se debe a que dichos análisis permiten obtener información significativa no solo sobre la naturaleza de las inclusiones no plásticas sino también sobre la selección y tratamiento de materias primas, los modos de elaboración de las piezas y, en este sentido, sobre las prácticas de producción, la identificación de tradiciones de manufacturas y posibles áreas de procedencia.

En el trabajo que inicia este Dossier, Isabelle Druc y Mauricio Uribe Rodríguez establecen el primer estudio petrográfico de la cerámica de varios pueblos aymaras del sector Isluga del altiplano de Tarapacá en el norte de Chile. La investigación incluyó alfarería de los estilos Inca Colonial, Etnográfica Colonial y Etnográfica Reciente, permitiendo distinguir las particularidades composicionales de las distintas aldeas. A su vez, logran diferenciar estas producciones con la manufactura de la región del valle de Tarapacá. 
En tanto, Nicolás Larcher y Beatriz Cremonte se centraron en el estudio microscópico de secciones delgadas combinado el análisis químico por Fluorescencia de Rayos X. El objetivo fue comprender las distintas producciones que presenta el estilo cerámico Inca Pacajes procedentes de varias regiones de los Andes Centro-Sur y determinar posibles áreas de manufactura a partir de la distribución geográfica del estilo, los agrupamientos definidos por el análisis de las pastas y las conexiones con las geologías locales.

En cambio, Sergio Vera y Guillermo de la Fuente presentan los resultados de los estudios realizados a una muestra cerámica de estilos Sanagasta, Inca y Diaguita Inca procedente del tambo incaico Costa de Reyes No5 (Catamarca, Argentina). De esta manera, obtuvieron una caracterización tecnológica del savoir faire propio de los alfareros para momentos de los períodos Tardío e Inca, observándose tendencias generales y cambios en las manufacturas alfareras en el sur del valle de Abaucán.

Por otra parte, Leticia Gasparotti investigó las elecciones tecnológicas de los alfareros del Período Tardío que habitaron el sitio Corral Alto ubicado en la quebrada del río Miriguaca en la microrregión de Antofagasta de la Sierra (Catamarca, Argentina). La información obtenida le permitió reflexionar sobre las prácticas alfareras como una producción local, no estandarizada y flexible y sus vinculaciones con otras manufacturas cerámicas de distintos sectores de dicha microrregión.

También, Vanesa Juarez fusiona los datos obtenidos del estudio petrográfico con los detalles tecno-morfológicos y funcionales de muestras cerámicas del Período Formativo recuperadas en contextos funerarios de los sitios Punta de la Peña 9- Sector III y Punta de la Peña 13- Sector I, localizados en la microrregión de Antofagasta de la Sierra. Esta visión integral le permitió generar interpretaciones acerca de los posibles criterios de selección seguidos por las poblaciones puneñas para la incorporación de dichos objetos en los contextos mortuorios.

Por su parte, Sebastián Carosio identificó particularidades tecnológicas que utilizaron los ceramistas en el área del sitio Tambería de Guandacol (La Rioja, Argentina). Su estudio se basó en los análisis petrográficos del estilo Sanagasta / Angualasto, cronológicamente asociado al periodo Tardío (ca. siglos XIII-XVII DC).

En tanto, Josefina Pérez Pieroni y Carlos Angiorama presentan los resultados del análisis de los materiales recuperados en un basurero del asentamiento de Moreta (Jujuy, Argentina), datado entre los siglos VII y XII de DC. Los autores buscaron caracterizar las tradiciones tecnológicas involucradas en la confección de los enseres cerámicos, a partir del 
estudio de la cadena operativa de producción y su comparación con casos contemporáneos disponibles en la cuenca de Pozuelos y otros sectores de la Puna de Jujuy.

Finalmente, nuestro trabajo indagó sobre los modos de hacer cerámica que tenían las sociedades prehispánicas de finales del primer milenio D.C. que habitaron el sur de los valles Calchaquíes y los valles de El Bolsón y Hualfín en el Noroeste argentino. El carácter macrorregional de la muestra posibilitó detectar circulación de piezas y regularidades de manufactura que permiten postular la presencia de un savoir faire de confección cerámica con particularidades locales.

\section{Bibliografía citada}

Cigliano, E.; Raffino, R. y H. Calandra

1972 Nuevos aportes para el conocimiento de las entidades alfareras más tempranas del Noroeste argentino. Relaciones de la Sociedad Argentina de Antropología VI: 225-236.

Convención Nacional de Antropología

1966 Primera Convención Nacional de Antropología (Primera Parte). Facultad de Filosofía y Humanidades, Universidad Nacional de Córdoba.

Cremonte, M.

1991 Caracterizaciones composicionales de las pastas cerámicas de los sitios Potrero Chaquiago e Ingenio del Arenal Médanos (Catamarca). Shincal 3: 33-47.

1996 Investigaciones Arqueológicas en la Quebrada de La Ciénega (Dpto. de Tafí, Tucumán). Tesis de Doctorado. Universidad Nacional de La Plata, La Plata.

Dougherty, B.

1975 Breve reseña sobre la arqueología del río San Francisco. Actas y Trabajos del Primer Congreso de Arqueología Argentina, pp. 363-382. Artes Gráficas, Buenos Aires.

González, A.

1964 La cultura de la Aguada del N. O. argentino. Revista del Instituto de Antropología II-III: 205-253.

González de Bonaveri, M.

1991 Tecnología de la cerámica arqueológica del Partido de Chascomús. La cadena operativa en el sitio La Guillerma 1. Arqueología 1: 105-124. 
Heredia, O.

1974 Investigaciones arqueológicas en el Sector Meridional de las Selvas Occidentales. Revista del Instituto de Antropología V: 73-132.

Krapovickas, P.

1975 Algunos tipos cerámicos de Yavi Chico. Actas y Trabajos del Primer Congreso de Arqueología Argentina, pp. 293-300. Artes Gráficas, Buenos Aires.

Linné, S.

1925 The Technique of South American Ceramics. Fjärde Följden Band 29, No 5. Elanders Boktryckeri Aktieboi, AG, Gotemburgo.

Núñez Regueiro, V.

1975 Cronología de los tipos cerámicos de los sitios de Alumbrera, Provincia de Catamarca (Culturas Alamito, Ciénaga y Condorhuasi). Actas y Trabajos del Primer Congreso de Arqueología Argentina, pp. 383-404. Artes Gráficas, Buenos Aires.

Shepard, A.

1985 [1956] Ceramic for the Archaeologists. Braun-Brumfield, Ann Arbor.

Tarragó, M.

1976 Alfarería típica de San Pedro de Atacama (Norte de Chile). Estudios Atacameños 4: 3773. 
